\title{
SUPPORTING CREATIVITY WITH VIRTUAL REALITY TECHNOLOGY
}

\author{
Graessler, Iris; Taplick, Patrick \\ Paderborn University - Heinz Nixdorf Institute - Chair for Product Creation
}

\begin{abstract}
This contribution includes the development and validation of a Virtual Reality (VR) supported creativity technique: "Sensory Stimulus Environment Technique". Key elements of this technique are the creativity process, a VR tool and the support of the tool (Virtual Creative Environments). The creativity process consists of phases for individual and group-based work. The VR tool "Virtual Creativity" includes functions to support the preparation of Virtual Creative Environments (VCE), the generation and evaluation of new ideas. For the generation of VCE, the tool possesses an environment configurator. Users of this function are supported by Design Rules for VCE.

For the validation of the creativity technique, it was used in a product engineering project. The project members procedure two phases of the creativity process (Preparation and Individual Idea Generation) and used "Virtual Creativity" to generate VCEs and ideas to solve their tasks. By questionnaires, functions of "Virtual Creativity" were assessed for generating VCEs and ideas.
\end{abstract}

Keywords: Creativity, Virtual reality, Innovation

\section{Contact:}

Taplick, Patrick

Paderborn University -Heinz Nixdorf Institute

Chair for Product Creation

Germany

patrick.taplick@hni.upb.de

Cite this article: Graessler, I., Taplick, P. (2019) 'Supporting Creativity with Virtual Reality Technology', in Proceedings of the 22nd International Conference on Engineering Design (ICED19), Delft, The Netherlands, 5-8 August 2019. DOI:10.1017/dsi.2019.207 


\section{INTRODUCTION}

Creativity is a human ability that enables development of innovative products. Amabile defines it as the production of solutions, responses and products for open tasks being characterized by the properties novelty and appropriateness (2012). Runco and Jaeger emphasize originality and effectiveness as important for creativity (2012). For achieving creativity in a structured way, creativity techniques are used. Workshops implementing creativity techniques are suitable to combine the generation of ideas and the advantages of collaboration. In case of distributed working teams, conventional creativity techniques such as brainstorming are hardly applicable (Rickards and DeCock 2012). The stationarity of such techniques leads to high monetary efforts and additional travel efforts for employees. An alternative is the usage of information and communication technology (ICT). Firstly, conventional ICT does not allow to interact intuitively with other project team members, secondly ICT is not adapted to structure and properties of creativity techniques being used in distributed working teams.

Virtual Reality (VR) technology provides the possibility of intuitive collaboration by immersion in a virtual environment and the usage of a virtual representation (avatar). To utilize the potentials of VR for fostering creativity, the technology has to be adapted for creativity techniques. In this case, the VR supported creativity technique "Sensory Stimulus Environment Technique" was developed. Main use cases of the integrated VR tool "Virtual Creativity" are determined by intuitive collaboration and support for idea generation by creating Virtual Creative Environments (VCE) (Graessler and Taplick 2018). VCEs are virtual environments (virtual scenes) consisting of a wide range of different virtual objects, textures and auditory files for stimulation. For implementing VR supported creativity techniques the following research question is essential: Which design guidelines and VR functionalities have to be provided to support the generation of VCEs for collaborative idea generation?

To answer this research question, a prototype based approach was chosen being necessary to gather direct feedback by potential users. The VR supported creativity technique "Sensory Stimulus Environment Technique" was developed and validated in a product engineering project. Its design was derived from cross-domain literature analysis in the fields of creativity, collaboration in distributed teams and VR technology (chapter 2). The framework for VR support (chapter 3) comprises the implementation of the creativity process (detailed in section 3.1), a VR tool supporting several phases of the creativity process (3.2) and the support (Virtual Creative Environments) for generating ideas which will be generated with the VR tool (3.3). Consequently, the paper at hand describes the development of the VR supported creativity technique "Sensory Stimulus Environment Technique" and its experimental validation (chapter 4 ) in a product engineering project.

\section{TECHNOLOGY SUPPORTED CREATIVITY}

The development of the VR supported creativity technique is based on literature research considering conventional ICT solutions and concepts for the support of creativity in distributed working teams. Additionally, first investigations of virtual environments for the support of creativity are described.

\subsection{Group creativity support platform}

Voigt and Bergener (2013) designed a framework of Group Creativity Support Systems (GCSS) including components for divergent and convergent working based on 13 design principles. Based on literature research, Wang and Nickerson identified specific approaches (e.g. support for problem identification and information retrieval) and relevant theories of creativity (e.g. factorial, associative) for the design of new individual creativity support systems. Gabriel et al. (2017) investigated the support of creative workshops by a GCSS. Guegan et al. (2016) considered the influence of represents (avatars) of engineers on creativity.

\subsection{Community supported creativity}

Global operating companies utilize community platforms for the generation and distribution of new ideas for solving problems and fulfil tasks. Albers et al. describe an approach implemented in an automotive company (2015). Another concept of Albers et al. is called ProVIL (2016): It is a virtual live lab including process and methods supporting distributed working teams while fulfilling their tasks. An innovation platform connects project team members, scientific supervisors and client. 


\subsection{Virtual reality supported approaches}

Lau and Lee (2012) focus on learning creativity and support of learning experiences at University. Experiments with highly interactive virtual environments indicated that fun and explorative factors are essential for creative virtual learning environments. Bhagwatwar et al. (2013) investigated the idea generation process of humans in creativity primed virtual environments. Priming describes a phenomenon of cognitive psychology. Alahuhta et al. (2014) identified eight affordances for the support of creativity by virtual environments by literature research. Characteristics of the VR technology such as immersion and rich visual information are identified as important aspects to support creativity.

Graessler et al. (2017) used six generated virtual environments in a first investigation differing by content, mobility and interaction (see figure 1). The six virtual environments differ by content and grade of possible mobility and interaction. Consequently, Graessler and Taplick (2018) investigated the efficiency of the creativity technique "Sensory Stimulus Environment Technique" supported by pre-developed virtual environments in comparison with two conventional techniques without technology support: brainstorming and "Sensory Picture Technique". To improve the VR supported creativity technique, a configurator for environments is essential to vary VCEs and create a high grade of stimulation.

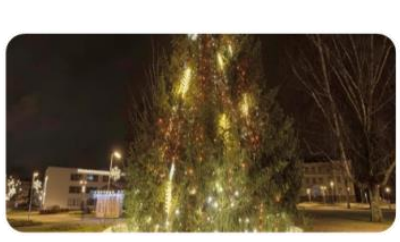

Content

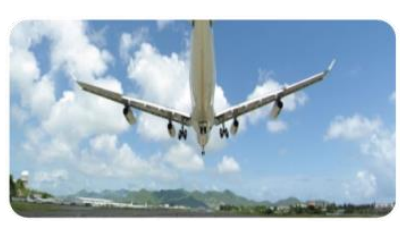

Figure 1. Used virtual creative environments.

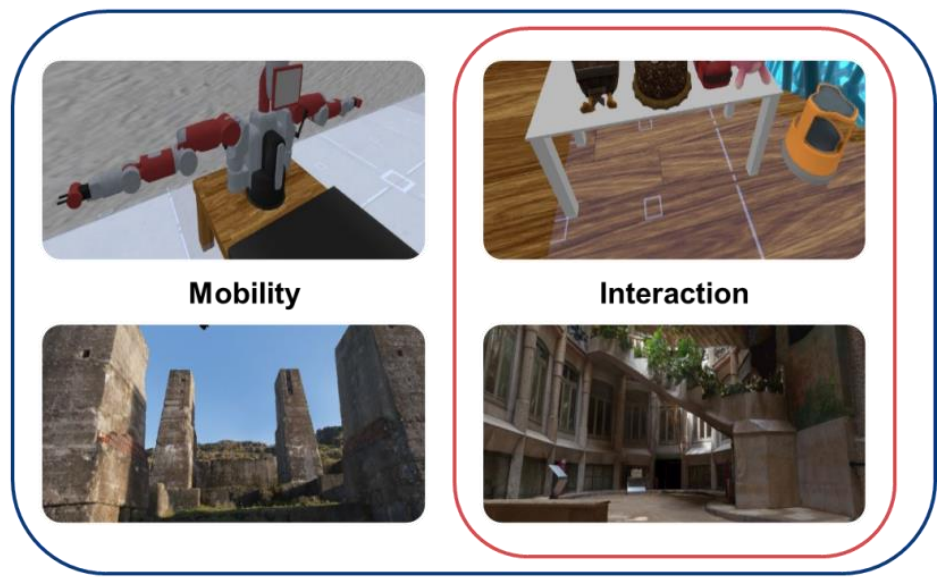

\section{FRAMEWORK OF A VIRTUAL REALITY CREATIVITY SUPPORT SYSTEM}

Based on literature research and preliminary investigations, the "Sensory Stimulus Environment Technique" was developed (Graessler, Taplick and Pottebaum 2017). The creativity technique is based on VR produced stimulation in VCEs. The stimulation of VCEs supports users with associations to generate ideas (Graessler and Taplick 2018). The VR tool "Virtual Creativity" is used for the design of VCEs and provides as a collaboration platform for teams. VR technology can be described by three dimensions: immersion, imagination and interaction (Burdea and Coiffet 1994). These three dimensions have an influence on creativity. User's imagination is elementary for being creative. The imagination is supported by the integration in virtual environments (immersion). The interaction of user and virtual environment supports the immersion. The following sections 3.1, 3.2 and 3.3 include the relevant elements for the design of a VR supported creativity technique: The creativity process, the supporting VR tool "Virtual Creativity" (Graessler and Taplick 2019) and the generated support of this technique. Figure 2 displays these elements and associated characteristics which have to be designed for the "Sensory Stimulus Environment Technique". The basis for the relevant elements builds design guidelines derived from the state of the art of creativity, distributed working teams and VR.

\subsection{Creativity process}

The creativity process consists of different phases being relevant to generate ideas and to solve problems from the identification of a task or problem to the assessment of generated ideas. Based on design guidelines for creativity processes, the creativity process includes phases for individual and group work. The design guidelines for the creativity process are divided into categories: "Process Phases", "Technology Implementation", "Functionalities", and "Methodical Support". 
In the context of the "Process Phases" the first step is the implementation of a task presentation for a common understanding of all project members and information basis. An important aspect is the implementation of divergent phases for the expansion of the idea space and convergent phases for the selection of the most suitable ideas. Stroeble and Diehl (1994) have determined that a first phase of an individual idea generation improves the group based idea generation. This sequence leads to a high diversity of ideas. The phase "Preparation" includes the identification of necessary information for the users, but also the generation of different virtual creative environments (VCE) by the moderator or project leader for the idea generation.
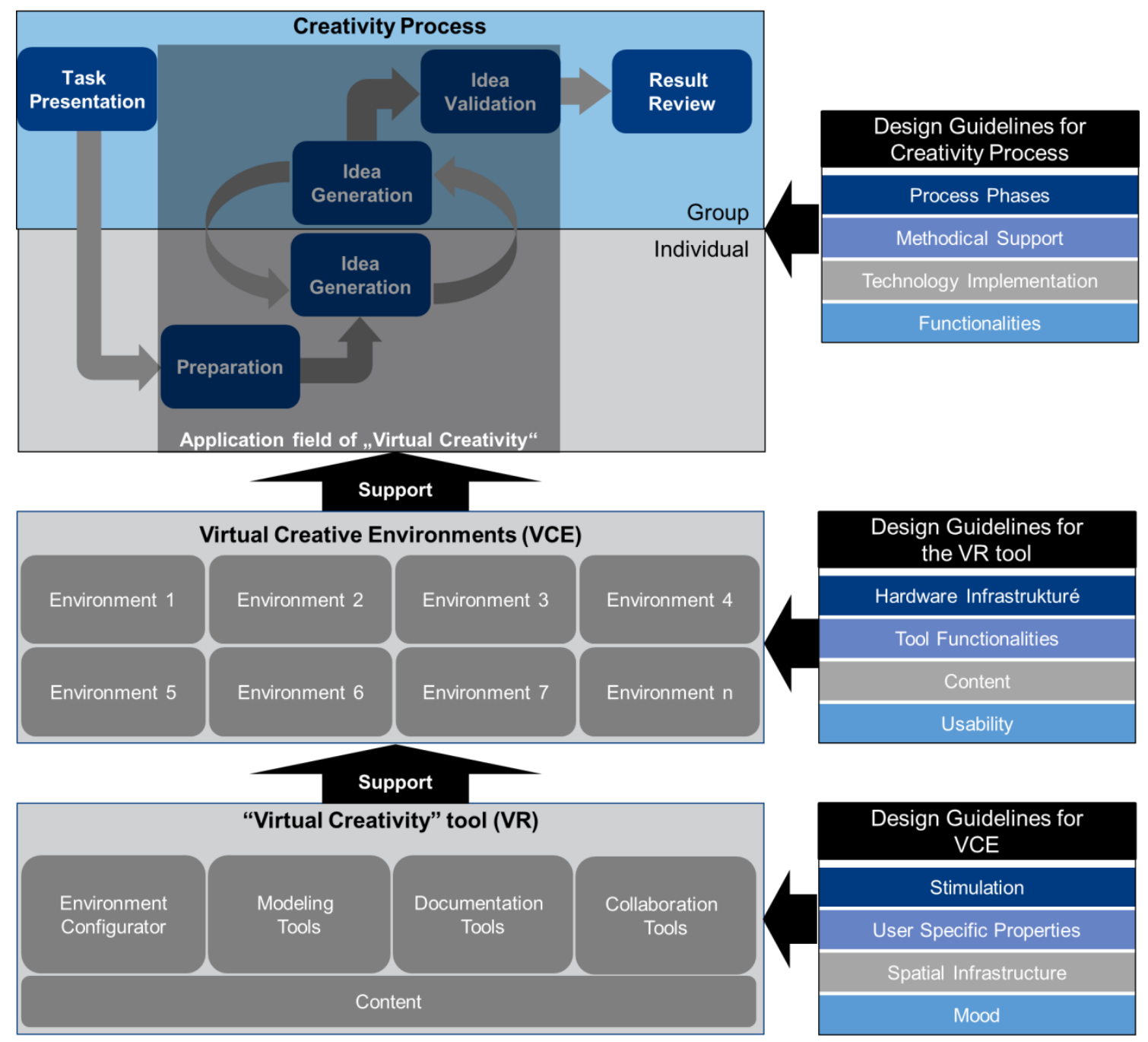

Figure 2. Concept of the "Sensory Stimulus Environment Technique".

In the category "Technology Implementation" of the design guidelines for creativity process, the usage of VR technology is focused. On the basis of preliminary studies on virtual environments for creativity (Alahuhta et al. (2014), Bhagwatwar et al. (2013)) and specific based on VR (Graessler and Taplick (2017)) the technology is a suitable possibility to stimulate human senses in idea generation. Especially, the immersion of users in virtual environments increases the visual perception. An additional benefit of the implementation of VR technology in the creativity process is the high degree of intuitive handling. Users are able to describe aspects with gestures generated with a natural user interface. Users experience working in the VCE resulting from the VR tool "Virtual Creativity" as user friendly. They learn its handling in few minutes. Intrinsic motivation of technology affine people can be stimulated. The usage of new technologies such as Virtual and Augmented Technology leads to an intrinsic motivation (Soler et al., 2017). But there are also challenges such as a suitable maturity of VR technology to be used by a majority of employees in a company.

The support of "Functionalities" for the creativity process is focused by handling of generated ideas with the help of functionalities by information systems. One functionality is the anonymization of 
generated ideas. It fosters sharing of ideas (Valacich et al. 1992). In companies and other institutions hierarchies play an important role. Employees of lower hierarchy levels may resolve their concerns to share ideas. For the processing of the generated ideas it is necessary to implement functionalities to document and track ideas. One aspect for the necessity of it is the gaining of more information in context of the generated idea. By tracking ideas the source for inspiration can be identified and used to understand the context of the generated idea.

In the context of the category "Methodical Support", a method has to be implemented to reduce the fixation on conventional ideas for solving a task. Users of the creativity technique have to consider several ways to create ideas for solving the task or problem. Possibilities are the generating of partial solutions or the imagination of the situation with the fulfilled solution. Additionally, the creation of a high number of ideas is important to find a suitable solution. The implementation of the "Path to Success" (P2S) method of Eppler (2012) supports the moderator in guiding the user in the creativity session. The influence of the moderator by using specific paths (partial ideas, actual state, desired state, situation with fulfilled solutions,...) helps users to regard the VCEs from different perspectives.

\subsection{Design guidelines for virtual reality tool "Virtual Creativity"}

The implementation of VR technology provides possibilities to support the creativity process. The phases of "Preparation", "Individual and Group Idea Generation" and the "Idea Validation" of the new "Sensory Stimulus Environment Technique" carry highest demands for support by VR (Figure 2). The phase "Preparation" includes the design of VCEs being necessary for the idea generation. For the phases of "Individual and Group Idea Generation", VR technology provides a benefit by dynamic visual stimuli. By visualization of generated ideas and discussions in a virtual room, VR technology can be used to support "Idea Validation". Design guidelines for VR tool are divided in the categories "Hardware Architecture", "Tool Functionalities", "Content" and "Usability". Using these guidelines, several necessary functions of the VR tool "Virtual Creativity" were identified.

According to the category "Hardware Architecture" the VR tool has to be supported by input and output hardware devices supporting several communication forms, interactions and different stimuli. For the creation of ideas users must have the possibility to interact with VCEs. Activities such as walking, teleporting and grabbing objects are essential for the interaction with these environments. The interaction within project teams have to be support by communication such as voice chat, gestures or text messaging. For the stimulation of human senses, different devices have to be part of the infrastructure. A suitable solution for input and output devices is the implementation of a Head Mounted Display (HMD) for the implementation of visual stimulation, communication forms and interactions. Devices such as the HTC Vive provide room-scaling for the mobility of users. Several scripts include functionalities to support teleportation for mobility and interaction with virtual objects. Voice chat and aural stimulation can be implemented with headsets for users.

The usage of olfactory and haptic stimulation is beneficial, but needs specific hardware such as force feedback gloves and masks for releasing smell. Another important topic is the integration of tools in specific networks. The tool has to work without failures and disruptions for the support of distributed working teams.

The category "Tool Functionalities" includes the functional integration in the VR tool. The focus of this category is the support of the idea generation phase in the creativity process. For the fulfilment of this task, the implementation of several tools is necessary. To generate different VCEs a configurator supported by a database with different content (being defined in the category "Content") is a suitable tool. The environment configurator includes scripts to import parameterized virtual objects (grade of complexity, topic, dimensions, ...). Another function of the configurator is the change of object textures and the skybox (background of a virtual environment). Modelling tools are required for the addition of visual aspects being not implemented in the database and the improvement of ideas by visual display. The usage of sketching tools supports users by creating objects leading to a visual stimulation or the visualization of a created idea. In the context of the idea visualization, a virtual whiteboard provides the possibility to improve ideas in groups. For the documentation of generated ideas tools resulting different medial content have to be implemented. Tools to generate screenshots and video clips with auditory input give user possibilities to review documented ideas. The last block of necessary tools is the support of the collaboration of teams in sessions. The implementation of avatars and scripts for auditory communication are essential parts to support collaboration. 
The category "Content" includes several types of artefacts being the fundament for tools such as configurator and modelling tools. Virtual objects, textures, materials and cube maps are content to vary the design of the VCEs. The content has to vary by topics to create several kinds of experience and moods. The implementation of scripts representing functionalities in a database makes the different configurations of virtual environments in context of interaction and mobility possible.

The category "Usability" impacts the design of the VR tool. Like the intuitive usability of the hardware, the software must include tools being designed for a user friendly usage. For this task conventional guidelines of organizations such as "Verein deutscher Ingenieure" (VDI) and VR specific recommendations of hardware and software developers exist.

\subsection{Design guidelines for virtual creative environments}

The creativity process and the implemented VR tool form the fundament for the stimulation by virtual content. The support of the VR tool for generating ideas are VCEs. The design guidelines for VCEs in combination with the environment configurator provide support for project leaders and moderators for designing VCEs for sessions. In section 3.2 different functions of the environment configurator (implementation of virtual objects, change of textures and backgrounds) are described. The design guidelines for VCEs consist of factors to foster creativity. The consideration of design guidelines while using the environment configurator supports the designer (in a project: moderator or project leader). The design guidelines are divided in the categories "Stimulation", "User Specific Properties", "Spatial Infrastructure" and "Mood".

The category "Stimulation" is essential according to the aim of the creativity technique "Sensory Stimulus Environment Technique", i.e., to create ideas by the stimulation by and association with virtual content. The virtual environments must include a wide range of different visual stimuli. The visual stimulation is created by the differentiation and combination of virtual objects, textures, materials and backgrounds. The different content should be used to model experiences, topics and moods. The additional implementation of tactile, olfactory and auditory stimuli supports the immersion and realism of a VCE. Sound, smell and touch can create associations leading to suitable ideas.

The second category of design guidelines focuses "User Specific Properties" in the context of creativity. The grade of complexity is one aspect which have to differ in the VCEs. The suitable grade of complexity depends on the user.

In the context of the "Spatial Infrastructure" VCEs should not include a high amount of spatial constraints leading to decreasing of creativity. The inclusion of areas for prototyping/modelling and the ability of mobility in VCEs are elements for the spatial layout which have to be considered. Contrary to stimulation by different stimuli areas with reduced stimulation can influence the creativity of users. Empty environments with a low amount of visual stimulation can be used to be filled with ideas by users with modelling and sketching tools.

The focus of the fourth category "Mood" lies on generating fun by playful and experimental atmosphere. The experience of fun supports higher acceptance by users. Additionally, it increases the intrinsic motivation of users to apply the technique and its VR implementation. Interaction with VCEs fosters the generation of associations leading to possible new ideas.

\section{VALIDATION}

The validation of the developed creativity technique was conducted by experiment. The "Sensory Stimulus Environment technique" was used in the product engineering project of the course "Integrated Product Engineering" at IPEK - The Institute of Product Engineering (Karlsruhe Institute of Technology). In the course the phases "Preparation" and "Individual Idea Generation" of the creativity process and the prototype of the VR tool "Virtual Creativity" were validated. The prototype includes functionalities to create VCEs. Additionally, users can create ideas in the phase of individual idea generation. For this phase, the prototype provides functionalities for the support of idea generation (collaboration, modelling and documentation).

\subsection{Validation design}

42 students acting in seven groups work in cooperation with a global acting company half a year to generate solutions for a specific task. Every student in group represents a role in the project (e.g. system engineer, validation engineer, ...). For the generation of solutions, the project teams pass 
different phases of a process (Albers et al. 2016). The developed creativity technique was used to generate product profiles for transportation alternatives for mining industries in a creativity workshop. The profiles include first ideas and constraints for the development of solutions. Figure 3 displays the procedure of the validation. Questionnaire 1 was designed to gather information in the context of experiences with VR technologies and creativity techniques before the validation. The first phase of the validation "Preparation" includes the generation of VCEs being executed by methods engineers (seven users). For the support of the 30 minutes session guidelines for the design of VCEs were considered before the Preparation phase. The method engineers, a role in the project groups, received a short handling introduction of the VR system. Afterwards, they used several functions such as the implementation of virtual objects of the environment configurator and the sketching tool. The implemented virtual objects were combined with several backgrounds based on different cube maps. Output of the phase "Preparation" were the created VCEs. Additionally, the methods engineers answered a questionnaire after the session focussing the necessity of the design guidelines for VCEs, function and usability of "Virtual Creativity". Based on the input in form of the generated VCEs and a presentation of the creativity technique at least two other indefinite roles of every group used "Virtual Creativity" for the individual idea generation (15 users).

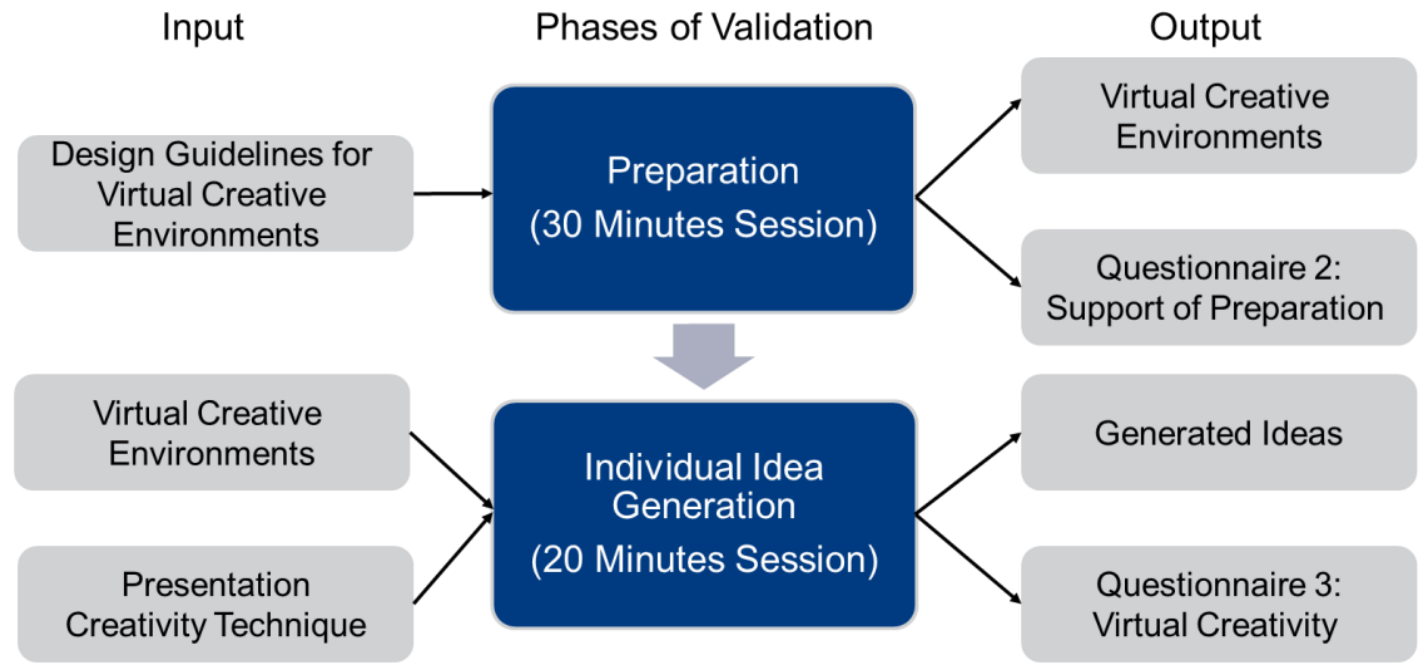

Figure 3. Procedure of validation.

The tool provided functions such as changing environments and a virtual whiteboard to support users in generating ideas. Every user was supported by a moderator which gave input by using aspects of the P2S method to identify ideas in the 20 minutes session. The users generated ideas for the product profile and answered a questionnaire dealing with the suitability to support the creativity techniques with different functionalities of the VR tool.

\subsection{Results}

Users had to answer three questionnaires to validate the suitability of VR tool functionalities to support designing VCEs and generate ideas. The first one focused on previous experiences with a) VR technology and b) creativity techniques. The second questionnaire included questions in the context of designing VCE with the design guidelines and the usage and usability of the VR tool. The design guidelines for VCEs are elementary to support designers of VCEs in the creativity technique. The third one dealt with the validation of design guidelines, applicability and usability of the VR tool in context of the idea generation. Additionally, users had the opportunity to mention improvement potentials and wishes for technique and tool.

\subsubsection{Questionnaires}

The first questionnaire on "Preliminary information" being answered by the students before the validation provides information in context of experiences with VR and creativity techniques. More than 95\% had theoretical knowledge (articles, pictures or other information of VR) or practical experiences (usage of a VR tool) with the technology. Only $22.2 \%$ of students mentioned that they use creativity techniques often. More than half of the students (52.8\%) use them sometimes and 25\% 
never use creativity techniques. One third of the students assessed themselves as very creative. More than the half think that they are personally partly creative. The motivation of the students in the project was very high $(69.4 \%)$ or high $(30.6 \%)$. The students mentioned several different aspects which have positive influence on their creativity: Music, silence, teamwork, freedom, positive mood, sketches.

The "Enjoyment" of using the technique and VR technology were answered in the second and third questionnaires. Every user for designing VCEs felt a high level of enjoyment (100\% fully). The users of the idea generation differ in using the technique (61.5\% fully, $38.5 \%$ partly).

In the second questionnaire on "Design guidelines" users assessed how they felt supported by eleven selected design guidelines (the other two guidelines are focused on virtual collaboration) for designing VCEs (figure 4). The users for the idea generation assessed the input of the design guidelines for generating ideas (scale 1 for weak to 4 for strong). Design guidelines 12 (enjoyment) and 13 (playful/experimental environment) were assessed as strong support for designing VCEs and generating ideas. The generation of new experiences (design guideline 4) was also assessed as a suitable support for both tasks. The assessment of design guidelines 1 (visual stimulation) and 9 (Areas for prototyping) differ for both tasks. The users designing VCEs felt design guidelines 1 and 9 as a strong support. Users using the tool for the idea generation think that both design guidelines are less important (design guideline 1: 2.5, design guideline 9: 2.2). Generally, users preparing the VCEs assessed the design guidelines more important than the group for the idea generation.

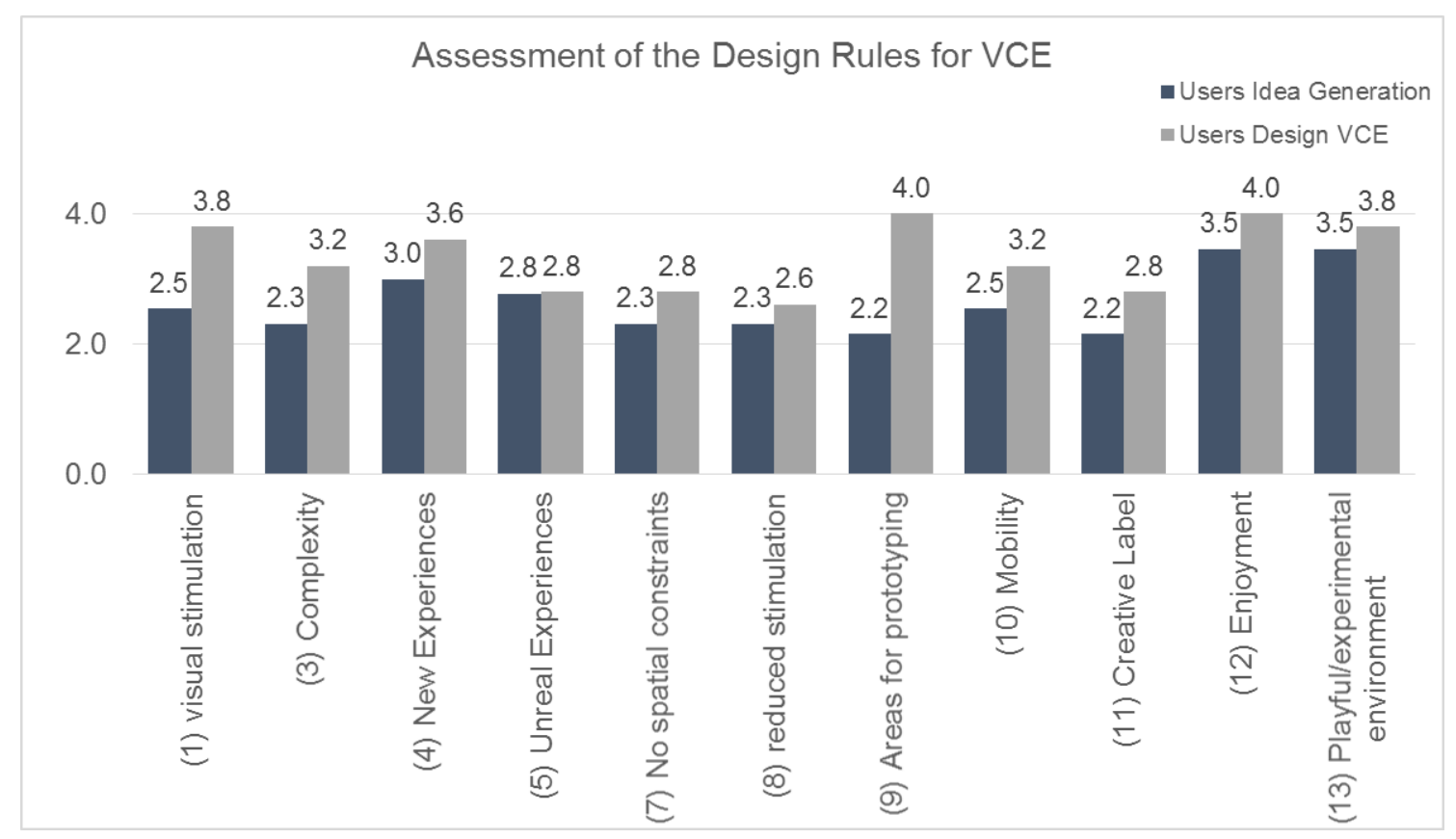

Figure 4. Assessment of design guidelines for VCEs.

To improve the approach of the "creativity technique" questions in the context of structure, moderator and interaction in VR were answered by all users. Users for designing VCEs wished more time to generate environments (80\%). Only $40 \%$ needed more support to design VCEs. The majority of these users felt suitably supported by the design guidelines.

Users with the task to generate ideas wished more team interaction $(46.1 \%)$ and a clearer structure of technique $(23.1 \%)$. Additionally, they wanted to have more time for the idea generation process. The implementation of the moderator with the tasks to support by showing other ways to create ideas was assessed positively by these users (fully: $53.8 \%$, partly: $30.8 \%$ ).

In the second and third questionnaire users had to assess "tool functions" of the VR tool "Virtual Creativity" to fulfil their task (scale 1 for weak to 4 for strong). The results are displayed in figure 5 . The functions Insert simple objects, insert complex objects and the sketching tool play an important role to design VCEs. Users for the generation of ideas were asked about additional functions: Virtual whiteboard, Changing environments and Load object configurations (combination and orientation of objects). The functions being relevant in the context to design VCEs are assessed with high values (3, 3.5 and 4.0). Especially, the function insert complex objects was assessed with the highest possible value. For users generating ideas the functions change environment, insert complex objects and load 
object configuration were assessed as important for the tasks (3.0, 3.15 and 3.15). Other functions such as sketching tool and virtual whiteboard achieved average values to generate ideas. For following phases of the creativity process (group based idea generation and idea validation) the different functions have to be check for suitability. Functions such as virtual whiteboard and the sketching tool can have a higher impact on the tasks in these phases. The usage of "Virtual Creativity" showed that it includes functions being relevant for design VCEs and generating ideas.

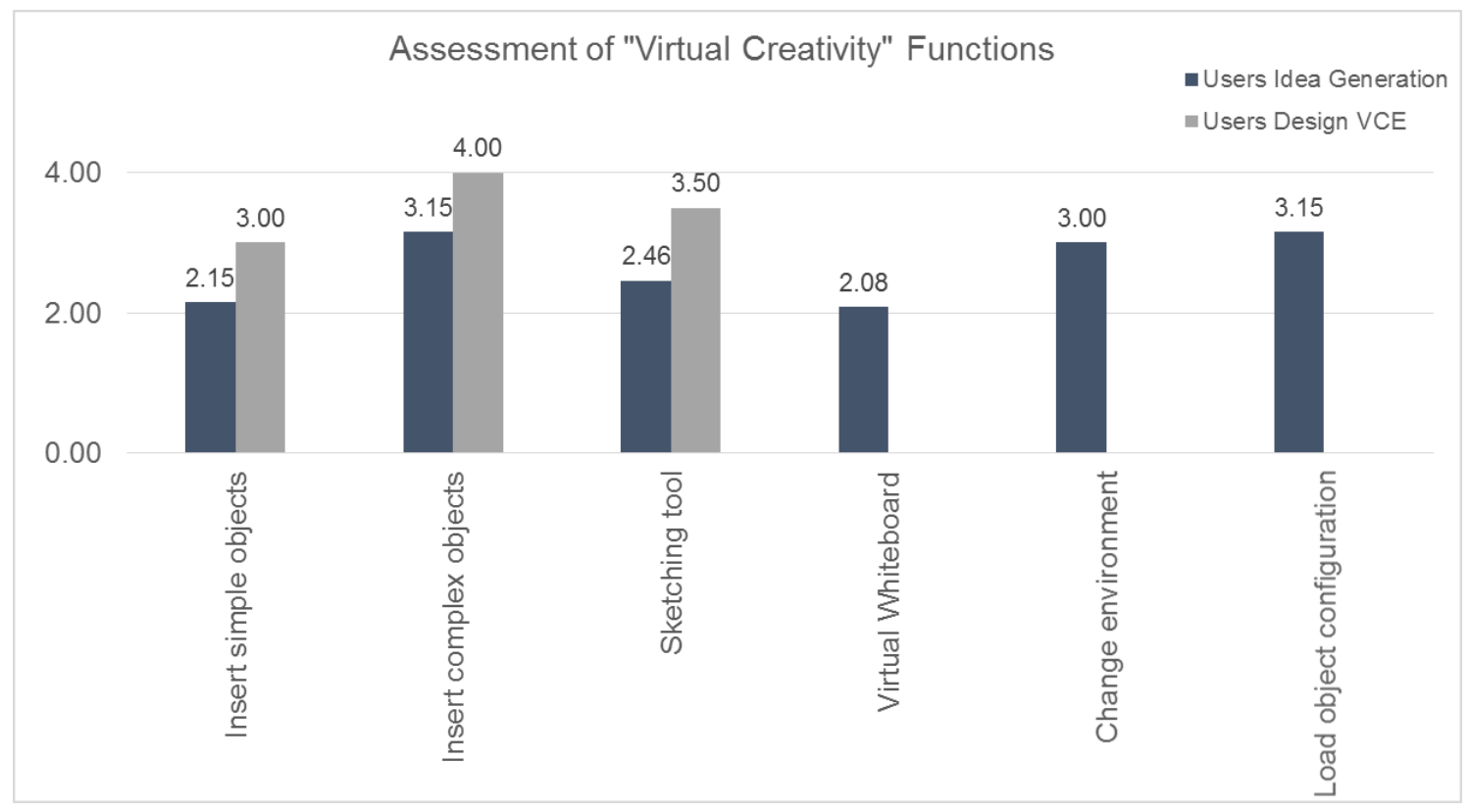

Figure 5. Assessment of "Virtual Creativity" functions.

\subsubsection{User-specific improvement potentials}

The second and third questionnaire included the demand for improvement potentials. For designing VCEs users wished for 3D models being relevant for the specific tasks in a project. Other users wished the possibility to interact with other users in the VCEs.

For users generating ideas the time was too short. An expansion of time or a limitless usage of the tool would be a solution to increase results. Additionally, a higher number of complex objects and dynamic objects are demands being relevant for the users.

\section{CONCLUSION AND OUTLOOK}

The creativity technique "Sensory Stimulus Environment Technique" based on a creativity process being supported by the VR tool "Virtual Creativity". The paper at hand focused on research questions regarding the generation of Virtual Creative Environments (VCE) and the generation of ideas. Which design guidelines and VR functionalities have to be provided to support these tasks? To answer these questions, a prototype based experimental methodology was applied. The prototype was validated in a product engineering project of students based on a real tasks of an industrial company. Results of the phases "Preparation" and "Individual Idea Generation" show that specific design guidelines (creating new experiences, enjoyment and playful/experimental atmosphere) support project leaders or moderators in creating virtual creative environments. Additionally, the two different user groups (methods engineers and indefinite roles) of the tool rated the relevancy of functionalities for designing VCEs and generating ideas. The suitability of functions is task related. Users designing VCEs rated the functions for inserting objects and sketching high. For the generation of ideas users preferred the usage of functions for changing environments and loading object configurations. Finally, users suggested further potentials for improvement of functions such as the implementation of additional functionalities to facilitate the usage of "Virtual Creativity" (e.g. button to load the same object several times in an environment). The integration of suitable functionalities in a VR tool and the implementation of support such as the design guidelines lead to a better support for distributed working teams. The generation of ideas in a collaborative virtual environment provides the reduction of costs and stress for companies and employees. 
The next step is the investigation of the phases "Group based Idea Generation" and "Idea Validation". After integrating a collaboration mode in "Virtual Creativity" the creativity technique will be validated in a distributed working project team in an industrial environment. Especially, the collaboration of team members in virtual environments will stand in the focus of research.

\section{REFERENCES}

Albers, A., Maul, L., Bursac, N. and Heismann, R. (2015), “Connected Creativity - A human centered Community Platform for Innovation Impulses", The Third International Conference on Design Creativity (3rd ICDC), 12th-14th January 2015, Bangalore, India.

Albers, A. Bursac, N., Walter, B., Hahn, C. and Schröder, J. (2016), "ProVIL - Produktentwicklung im virtuellen Ideenlabor", Entwerfen Entwickeln Erleben 2016, Dresden.

Alahuhta, P., Nordbäck, E., Sivunen, A. and Surakka, T. (2014), "Fostering Team Creativity in Virtual Worlds", Journal of Virtual Worlds Research Volume 7, Number 3, Lantern (2), September, 2014.

Amabile, T. M. (1996), "Creativity and innovation in organizations”. Harvard Business School

Bhagwatwar, A., Massey, A.P. and Dennis, A.R. (2013), "Creative Virtual Environments: Effect of Supraliminal Priming on Team Brainstorming”, 46th Hawaii International Conference on System Sciences.

Burdea, G. and Coiffet, P. (2003), "Virtual reality technology", 2nd ed., Wiley, Hoboken, NJ.

Eppler, M. J. and Pfister, R. A. (2012), "Paths to Success: A Sketch-based Creativity Technique for Individuals and Teams", 16th International Conference on Information Visualisation, Washington, D.C., USA.

Gabriel, A., Monticolo, D., Camargo, M. and Bourgault, M. (2017), “Conceptual Framework of an Intelligent System to Support Creative Workshops”, Springer International Publishing AG 261 D. Cavallucci (ed.), TRIZ - The Theory of Inventive Problem Solving.

Graessler, I., Taplick, P. and Pottebaum, J. (2017), "Enhancing Innovation Processes by Disruptive Technologies", in: SCIFI-IT 2017, S. 19-26, 2017 EUROSIS, EUROSIS-ETI.

Graessler, I. and Taplick, P. (2018), "Virtual Reality unterstützte Kreativitätstechnik: Vergleich mit klassischen Techniken”, in: Krause, D., Paetzold, K. and Wartzack, S. (Hrsg.) Design for X - Beiträge zum 29. DfXSymposium, S. pp. 215-226, Sep. 2018.

Graessler, I. and Taplick, P. (2019), "Architecture of a Virtual Reality-based Tool for the Support of Creativity", in: SCIFI-IT 2019, EUROSIS.

Guegan, J., Buisine, S., Mantelet, F., Maranzana, N. and Segonds, F. (2016), “Avatar-mediated creativity: When embodying inventors makes engineers more creative", in Computers in Human Behavior 61 pp. 165-175.

Lau, K. W. and Lee, P. Y. (2012), "The use of virtual reality for creating unusual environmental stimulation to motivate students to explore creative ideas", in Interactive Learning Environments, 2015, Vol. 23 No. 1, pp. 3-18.

Rickards, T. and DeCock, C. (2012), "Understanding organizational creativity: Toward a multiparadigmatic approach”, in M. A. Runco (Ed.), Creativity Research Handbook (Vol. 2, pp. 1-32), Hampton Press, Cresskill, NJ.

Runco, M. A. and Jaeger, G. J. (2012), “The Standard Definition of Creativity”, Creativity Research Journal Vol. 24, pp. 92-96.

Soler, J. L., Ferreira, J., Contero, M. and Alcañiz, M. (2017), “The Power of Sight: Using Eye Tracking to assess Learning Experience (LX) in Virtual Reality Environments", Proceedings of the 11th International Technology, Education and Development Conference 2017.

Stroebe, W. and Diehl, M (1994), "Why groups are less effective than their members: on productivity losses in idea-generating groups”, European review of social psychology, 1994, Vol. 5 No. 1, pp. 271-303.

Valacich, J. S., Jessup, L. M., Dennis, A. R. and Nunamaker, J. F. (1992), “A conceptual framework of anonymity in group support systems”, Group Decision and Negotiation, Vol. 1 No. 3, pp. 219-241, 1992.

Voigt, M. and Bergener, K. (2013), "Enhancing Creativity in Groups - Proposition of an Integrated Framework for Designing Group Creativity Support Systems", 46th Hawaii International Conference on System Sciences.

Wang, K. and Nickerson J.V. (2017), “A literature review on individual creativity support systems”, in Computers in Human Behavior Vol. 74 pp. 139-151.

\section{ACKNOWLEDGMENTS}

We would like to thank the project teams and the scientific supervisors of the IP project at IPEK - The Institute of Product Engineering (Karlsruhe Institute of Technology) for the excellent collaboration on overarching topics integrating the "Sensory Stimulus Environment Technique". 